\title{
Criminologie
}

\section{La judiciarisation internationale des criminels de guerre : la solution aux violations graves du droit international humanitaire?}

\section{Lison Néel}

Volume 33, numéro 2, automne 2000

Problèmes sociaux et système pénal

URI : https://id.erudit.org/iderudit/004737ar

DOI : https://doi.org/10.7202/004737ar

Aller au sommaire du numéro

\section{Éditeur(s)}

Les Presses de l'Université de Montréal

ISSN

0316-0041 (imprimé)

1492-1367 (numérique)

Découvrir la revue

Citer cet article

Néel, L. (2000). La judiciarisation internationale des criminels de guerre : la solution aux violations graves du droit international humanitaire? Criminologie, 33(2), 151-181. https://doi.org/10.7202/004737ar
Résumé de l'article

Les Nations Unies ont doté peu à peu la communauté internationale de traités, de conventions ou de principes engageant la responsabilité pénale internationale des individus pour permettre l'amélioration du respect des droits de l'homme les plus fondamentaux, afin d'éviter à tous les atrocités, la torture ou l'arbitraire. Malgré ces mesures de mise en œuvre très diversifiées, des violations graves continuent chaque jour à se dérouler un peu partout dans le monde. La communauté internationale ne réagit que lorsqu'elles atteignent un caractère massif, systématique et insupportable. L'échec de ces mécanismes de mise en œuvre a été le point de départ de la mise sur pied d'une justice pénale internationale. La judiciarisation internationale des criminels de guerre par le biais des tribunaux pénaux internationaux ad hoc ou de la cour pénale internationale permanente saura-t-elle pallier les carences et les faiblesses des systèmes pénaux nationaux quant à la répression des criminels de guerre ?
Ce document est protégé par la loi sur le droit d'auteur. L'utilisation des services d’Érudit (y compris la reproduction) est assujettie à sa politique d'utilisation que vous pouvez consulter en ligne.

https://apropos.erudit.org/fr/usagers/politique-dutilisation/ 


\title{
La judiciarisation internationale des criminels de guerre : la solution aux violations graves du droit international humanitaire?
}

\author{
Me Lison Néel ${ }^{1}$ \\ Candidate au doctorat \\ Faculté de droit \\ Université de Montréal \\ neell@magellan.umontreal.ca
}

RÉSUMÉ - Les Nations Unies ont doté peu à peu la communauté internationale de traités, de conventions ou de principes engageant la responsabilité pénale internationale des individus pour permettre l'amélioration du respect des droits de l'homme les plus fondamentaux, afin d'éviter à tous les atrocités, la torture ou l'arbitraire. Malgré ces mesures de mise en œuvre très diversifiées, des violations graves continuent chaque jour à se dérouler un peu partout dans le monde. La communauté internationale ne réagit que lorsqu'elles atteignent un caractère massif, systématique et insupportable. L'échec de ces mécanismes de mise en œuvre a été le point de départ de la mise sur pied d'une justice pénale internationale. La judiciarisation internationale des criminels de guerre par le biais des tribunaux pénaux internationaux ad hoc ou de la cour pénale internationale permanente saura-t-elle pallier les carences et les faiblesses des systèmes pénaux nationaux quant à la répression des criminels de guerre?

ABSTRACT - The United Nations has gradually endowed the international community with treaties, agreements or principles engaging the international penal responsibility of individuals, in order to reinforce the respect of the most fundamental human rights and to avoid atrocities, torture or arbitration. Despite these very diversified measures, serious violations keep appearing every day all over the world. The international community only reacts when these violations reach massive, systematic and unbearable proportions. In order to correct the failures of theses mechanisms, an international

1. L'auteure tient à remercier le Fonds pour la formation de chercheurs et l'aide à la recherche (FCAR) ainsi que la Faculté de droit et la Faculté des études supérieures de l'Université de Montréal pour leur soutient financier. 
penal justice was established. Will the international "judiciarisation" of the war criminals through the international penal courts "ad hoc" or through the permanent International Criminal Court be able to compensate for the deficiencies and weaknesses of the national penal systems in the repression of war criminals?

Il ne peut y avoir de paix sans justice, ni de justice sans loi, ni de loi digne de ce nom sans un tribunal chargé de décider ce qui est juste et légal dans des circonstances données.

Benjamin Ferencz, ancien procureur au tribunal de Nuremberg

\section{Introduction}

$\mathrm{Au} \mathrm{XX} \mathrm{X}^{\mathrm{e}}$ siècle, les droits de l'homme les plus fondamentaux occupent une place de choix dans les relations internationales. Les horreurs de la Deuxième Guerre mondiale et l'impunité des responsables des violations graves au droit international humanitaire ont attiré l'attention de l'opinion publique de la société civile internationale sur la nécessité de faire respecter ces droits fondamentaux.

Malgré la codification de plusieurs normes de protection des droits fondamentaux dans les instruments internationaux et de l'introduction de la responsabilité pénale internationale de l'individu, l'effectivité de cette protection, en droit interne et en droit international, s'est heurtée à l'hostilité des États tentant de préserver à tout prix leur souveraineté en matière pénale.

Cette dichotomie est présente depuis longtemps et s'est accrue ces dernières années. En effet, la communauté internationale s'est dotée depuis la Seconde Guerre mondiale d'une série d'instruments internationaux ${ }^{2}$, de tribunaux internationaux pénaux $a d b o c^{3}$, et d'une

2. Par exemple: Convention pour la prévention et la répression du crime de génocide, 11 décembre 1946, 78 R.T.N.U. 277; Convention de Genève pour l'amélioration du sort des blessés et des malades dans les armées en campagne, 12 août 1949, 75 R.T.N.U. 31; Convention de Genève pour l'amélioration du sort des prisonniers de guerre, 12 août 1949, 75 R.T.N.U. 135; Convention de Genève pour l'amélioration du sort des blessés et malades et des naufragés des forces armées sur terre, 12 août 1949, 75 R.T.N.U. 85; Convention de Genève pour la protection des personnes civiles en temps de guerre, 12 août 1949, 75 R.T.N.U. 287; Convention internationale sur l'élimination et la répression du crime d'apartheid, 30 novembre 1973, 1015 R.T.N.U. 243; Protocole additionnel aux Conventions de Genève du 12 août 1949 relatif à la protection des victimes de conflits armés internationaux, 12 décembre 1977, 1125 R.T.N.U. 3; Protocole additionnel aux Conventions de Genève du 12 août 1949 relatif à la protection des victimes de conflits armés non internationaux, 12 décembre 1977, 1125 R.T.N.U. 609; Convention contre la torture et autres peines ou traitements cruels, inbumains ou dégradants, 9 décembre 1984, AG NU A/rés./39/46.

3. Accord de Londres, 8 août 1945, 82 R.T.N.U. 279 (ci-après Statut de Nuremberg); Charte du Tribunal international pour l'Extrême-Orient, Déclaration du Commandant suprême des Forces alliées 
cour pénale internationale permanente ${ }^{4}$ et, simultanément, elle reste toujours consternée devant la multiplication des conflits intra-étatiques et les atrocités commises dans ces situations où le recours systématique à la force sert encore à subjuguer les populations.

Nous allons, dans un premier temps, suivre historiquement la création des normes internationales qui ont amené la justice pénale internationale et, ensuite, analyser les limites de cette judiciarisation internationale.

\section{Une avancée pour le respect des droits fondamentaux}

L'émergence de la responsabilité pénale internationale des individus se situe essentiellement au $\mathrm{XX}^{\mathrm{e}}$ siècle dans le droit des conflits armés (Pictet, 1986)5. La fin de la Première Guerre mondiale est le point de départ d'une justice pénale internationale qui a atteint son but ultime, le 17 juillet 1998 , après cinq semaines de négociations entre les représentants de 159 États : l'adoption du Statut de Rome par la Conférence des Nations Unies sur la création d'une cour criminelle internationale permanente.

Nous traiterons, tout d'abord, du développement de la responsabilité pénale internationale de l'individu sous l'égide de la Société des Nations et de l'Organisation des Nations Unies en suivant l'émergence de ce principe jusqu'à son effectivité par la création des tribunaux militaires de Nuremberg et de Tokyo, tout en soulignant l'apport de deux réalisations importantes pour le droit international humanitaire. Malgré l'instauration de ces normes de protection des droits fondamentaux, le principe

en Extrême-Orient, 19 janvier 1946, Mémorandum du Secrétaire général, A/CN.4/5, 1949 (ci-après Statut de Tokyo); Statut du Tribunal pénal international pour l'ex-Yougoslavie, Rapport du Secrétaire général établi conformément au paragraphe 2 de la résolution 808 (1993) du Conseil de sécurité, 3 mai 1993, S/25704 (ci-après Statut du Tribunal pour l'ex-Yougoslavie); et Statut du Tribunal international pour le Rwanda, annexé à la Résolution 955, Conseil de sécurité, 8 novembre 1994 (ci-après Statut du Tribunal pour le Rwanda).

4. Conférence diplomatique de plénipotentiaires des Nations, Statut de Rome de la cour pénale internationale, A/CONF.183/9, 17 juillet 1998 (ci-après Statut de Rome).

5. Le droit international humanitaire, appelé aussi droit des conflits armés, a pour objectif de réglementer les hostilités afin d'en atténuer les rigueurs. Le droit international humanitaire se compose $\mathrm{du}$ « droit de Genève » et « du droit de La Haye ». Le droit de Genève, dit droit humanitaire, tend à sauvegarder les militaires mis hors de combat et les personnes qui ne participent pas aux hostilités. Il donne la «primauté à l'homme et aux principes d'humanité ». Le droit de La Haye, dit droit de la guerre, détermine les droits et devoirs des belligérants dans la conduite des hostilités et restreint le choix des moyens de nuire. Le droit international humanitaire est donc un droit d'exception. Il s'applique lorsque beaucoup de règles cessent de s'appliquer. Il est l'ultime rempart contre la barbarie de la guerre. 
fondamental en droit international de la souveraineté des États servira à mettre en place une impunité de fait ou de droit des criminels de guerre. Deuxièmement, nous traiterons de la création et de la reconnaissance de trois des grandes infractions pénales internationales, soit les crimes de guerre, les crimes contre l'humanité et les crimes de génocide.

Les premières manifestations de la responsabilité pénale internationale des individus

Aux lendemains de la Première Guerre mondiale, l'idée d'une judiciarisation pénale internationale est lancée. La communauté internationale souhaite voir les responsables de crimes contre l'humanité et de crimes de guerre, commis au cours de la guerre 1914-1918, jugés par un tribunal international parce que « l'effet moral des mesures de répressions serait plus profond si elles étaient prises sur le plan international plutôt que par les États vainqueurs » (Assemblée générale, Commission du droit international (AG CDI), $1949: 2$ ).

De Versailles à Nuremberg

La Conférence de la paix, tenue à Paris en 1919, visait le jugement des accusés ressortissants des Puissances vaincues par un tribunal international. Elle créa la Commission des responsabilités des auteurs de la guerre et sanctions qui était chargée, notamment, de faire rapport sur la constitution d'un tribunal qui jugera les personnes responsables des violations des lois et coutumes de la guerre. Dans son rapport, la Commission recommandait la création d'un tribunal international, malgré les principes de droit international donnant autorité à tout État pour juger les individus accusés de crimes de guerre, afin de statuer sur les accusations de crimes commis contre des personnes de nationalités différentes et d'appliquer « les principes du droit des gens, tel qu'il résulte des usages établis entre nations civilisées, des lois de l'humanité et des exigences de la conscience publique » (AG CDI, 1949 : 51-55). La principale objection à la création de ce tribunal était motivée par l'incertitude du droit international quant à la détermination de la responsabilité des chefs d'État en cas de violations des « lois d'humanité » (AG CDI, 1949 : 56-64).

Les recommandations de la Commission ne furent pas adoptées par la Conférence de la paix. En juin 1919, le Traité de Versailles prévoyait seulement que l'ex-chef de l'État allemand, Guillaume II, serait jugé par un tribunal international " pour offense suprême contre la morale internationale et l'autorité sacrée des traités » (art. 227). Il prévoyait aussi que 
l'Allemagne devait remettre aux Alliés les accusés de crimes de guerre (art. 228) afin qu'ils soient jugés par des tribunaux militaires constitués par l'une des puissances alliées ou par des tribunaux militaires constitués de plusieurs puissances alliées (art. 229). En définitive, ces dispositions du Traité de Versailles ne furent pas appliquées : le Kaiser Guillaume II ne fut jamais jugé, puisque les Pays-Bas refusèrent de l'extrader et que l'Allemagne refusa d'extrader ses ressortissants (Taylor, 1995 : 26).

Suite à cet échec, le Conseil de la Société des Nations décida, en février 1920, la création du Comité consultatif de juristes chargé de préparer un projet pour l'établissement d'une cour permanente de justice internationale. L'instauration de cette juridiction fut reportée, car l'Assemblée considéra qu'elle était prématurée et qu'il était d'usage de laisser aux tribunaux nationaux la poursuite de ces crimes (AG CDI, 1949 : 10). Malgré cette mise au rancart, trois conférences internationales ${ }^{6}$ reconnurent qu'une collectivité internationale, basée sur les principes d'ordre et de justice, ne pouvait exister sans moyens de contrainte et de répression.

La Société des Nations entreprit, suite à ces travaux, de réexaminer la question de la création d'une cour pénale internationale afin d'assurer une répression efficace des crimes politiques et d'élaborer une convention internationale sur la répression du terrorisme. Le projet de convention prévoyait que les États, parties à celle-ci, devaient adopter dans leur législation nationale l'infraction qualifiant de criminels divers actes de terrorisme et la création d'une cour pénale internationale permanente pouvant juger les individus, déférés par les États, accusés d'une infraction prévue à la Convention pour la prévention et la répression du terrorisme (Pella, 1964 : 157-166). Au lieu d'envisager le problème de la justice internationale dans toute son étendue, soit la répression des crimes de guerre et des crimes contre l'humanité, la communauté internationale préféra se limiter à la répression du terrorisme. Ce projet de convention n'entra jamais en vigueur, parce que c'était le début de la Deuxième Guerre mondiale et que les États étaient réticents à se départir d'un peu de leur souveraineté dans le domaine pénal.

Ainsi, malgré le travail de tous ces comités, commissions et des différents projets de la Société des Nations et des États membres, aucun consensus et aucun instrument juridique n'exprime la volonté de la communauté internationale de réprimer pénalement les crimes internationaux à la veille du deuxième conflit mondial.

6. Ces conférences ont été organisées par l'Association de droit international (1922), l'Union parlementaire (1925) et le premier Congrès international de droit pénal (1926). 
Durant le conflit de 1939-1945, les crimes atroces, autant par leur dimension que par leur nature, commis par les nazis, ont imposé la réalisation pratique de l'idée d'une juridiction internationale pénale. Les Puissances alliées ont déclaré à plusieurs reprises leur intention de traduire en justice les coupables de crimes de guerre et d'autres atrocités dénommées plus tard « crimes contre l'humanité » (Pella, 1964 : 19-23).

Le 30 octobre 1943, les Puissances alliées exposent leur politique concernant les criminels de guerre allemands dans la Déclaration de Moscou : ils seront jugés et leur responsabilité pénale individuelle reconnue $^{8}$. Elle préconisait, pour la majorité des criminels de guerre, leur jugement par les tribunaux nationaux où les crimes avaient été commis. Elle prévoyait aussi que cette politique était « sans préjudice du cas des criminels allemands dont les crimes ne peuvent être situés en un endroit particulier et qui seront punis par une décision commune des gouvernements alliés » : la création d'une juridiction pénale internationale.

Deux tribunaux militaires internationaux furent institués pour juger les criminels de guerre nazis et japonais : le tribunal militaire international de Nuremberg et le tribunal militaire international pour l'Extrême-Orient. Le premier fut créé par l'Accord de Londres du 8 août 1945 et a siégé à Nuremberg, et le second fut créé par la Déclaration du 19 janvier 1946 et a siégé à Tokyo. Les procès portèrent sur la nature de la responsabilité pénale, soit la responsabilité individuelle pour les trois catégories de crimes prévus : les crimes contre la paix, les crimes de guerre et les crimes contre l'humanité ${ }^{\text {. }}$.

Comme dans le cas de la Première Guerre mondiale, la communauté internationale réagit, lors de la Deuxième Guerre mondiale, aux besoins de la cause et à la pression populaire : la nécessité fit loi. En effet, elle a considéré le passé et non l'avenir. Ce fut aussi le début de la justice pénale internationale ad hoc pour la répression des crimes contre la paix, appelés aujourd'hui les crimes d'agression, et pour celle des crimes de guerre et des crimes contre l'humanité.

7. Rappelons à ce sujet certaines déclarations : «l'appel à la conscience mondiale » lancé par les gouvernements français, britannique et polonais accusant l'Allemagne d'atrocités et de violations au droit des gens en Pologne; les déclarations de Churchill et de Roosevelt ( 25 octobre 1941) et de Molotov (27 novembre 1941 et 6 janvier 1942) au sujet des crimes de guerre; la Déclaration du Palais de Saint-James (13 janvier 1942) affirmant que « parmi les principaux buts de la guerre, le châtiment, par les voies d'une justice organisée, des coupables ou responsables de crimes de guerre qu'ils les aient ordonnés, perpétrés ou qu'ils y aient participé ».

8. Déclarations de Churchill, Roosevelt et Staline, Moscou, 30 octobre 1943.

9. Article 6 du Statut du Tribunal de Nuremberg et article 5 du Statut du Tribunal de Tokyo. 
Nuremberg a cependant été beaucoup critiqué et à juste titre. C'était le procès où les vainqueurs jugeaient les vaincus. Il ne présentait pas de suffisantes garanties d'objectivité. La juridiction était rétroactive et contrevenait au principe universellement accepté : nulla pona sine lege. Le tribunal s'en est justifié en soumettant que la juridiction avait intégré les éléments du droit coutumier et du droit naturel et ce, afin d'éviter l'aberration de l'impunité.

Le tribunal de Nuremberg émane exclusivement des États se considérant lésés par les agissements des criminels de guerre. Ces États ont créé la loi, assuré la poursuite et l'instruction, prononcé le jugement et exécuté les peines. Le tribunal de Nuremberg était une juridiction pénale internationale $a d$ hoc à laquelle chacun des États alliés, ayant appréhendé les criminels de guerre ennemis, remettait ses pouvoirs juridictionnels ${ }^{10}$. La Deuxième Guerre mondiale a convaincu de la nécessité d'organiser la société internationale afin d'éviter le retour de semblables crimes.

Suite aux critiques adressées à la justice ad hoc de Nuremberg et de Tokyo, les Nations Unies examinèrent le problème de l'instauration d'une juridiction pénale internationale permanente ainsi que la possibilité de codifier les principes de droit reconnus dans les statuts des deux tribunaux militaires internationaux et dans leurs jugements. L'Assemblée générale des Nations Unies a confirmé le 11 décembre 1946 « les principes de droit international reconnus par le Statut du tribunal de Nuremberg et par le jugement de celui-ci » pour leur donner une valeur permanente ${ }^{11}$.

La création du tribunal de Nuremberg a aussi été une formidable innovation. Il a réussi là où, lors de la Première Guerre mondiale, la communauté internationale avait échoué. Pour la première fois, la responsabilité personnelle d'un individu était mise en cause devant un tribunal répressif international et l'acte d'État comme fait justificatif des crimes commis était écarté. Le tribunal et le jugement de Nuremberg furent à l'origine d'un nouveau droit international : le droit pénal international (Taylor, 1995; Wieviorka, 1995). À Nuremberg une nouvelle incrimination fut définie : le crime contre l'humanité. Ce crime devenait une infraction internationale.

10. La présence de l'Union soviétique parmi les puissances accusatrices a donné un argument aux adversaires du procès. Elle avait signé avec l'Allemagne un pacte de nonagression, assorti d'un protocole partageant la Pologne, et avait perpétré aussi des massacres à grande échelle, notamment celui des officiers polonais à Katyn. L'Union soviétique se retrouva juge et, par le fait même, ses crimes furent éliminés.

11. Résolution 94(1), Assemblée générale, Nations Unies, 11 décembre 1946. 
Nuremberg a été une avancée innovatrice de la répression pénale internationale des criminels de guerre et de nombreux principes du droit international humanitaire y ont été consolidés. De plus, il a posé les jalons du droit international pour la répression du génocide. Malgré tout, Nuremberg fut une déception. L'espoir était grand après que les grands criminels furent jugés et punis que les autres le seraient aussi. Aujourd'hui, nous savons que beaucoup de ces criminels de guerre ne le furent jamais.

Nuremberg n'a donc été que le droit d'un moment et non pas l'application d'un droit préexistant. Le tribunal de Nuremberg était une juridiction d'exception, créé pour juger des infractions commises par certaines personnes et dans un temps déterminé. Le droit applicable, comme le tribunal, a été un droit ad hoc: le Statuténonçait non seulement le fonctionnement du tribunal, mais aussi le droit qu'il devait appliquer (Lombois, 1979 : 157). Étant donné la nature ad hoc du tribunal et du droit de Nuremberg, les Nations Unies ont senti la nécessité de proclamer que les principes du droit de Nuremberg seraient, pour l'avenir, le droit en vigueur. Cette reconnaissance est suffisante pour reconnaître le caractère international du droit pénal de Nuremberg. Mais qu'en est-il de la volonté de la communauté internationale ? Il faut reconnaître qu'elle n'a pas été tellement soutenue : ni la codification des infractions internationales ni la création d'une juridiction criminelle internationale permanente ne furent menées à terme. Le droit de Nuremberg fut donc relégué à une simple nécessité due à l'époque.

\section{Deux réalisations importantes}

En l'absence d'une juridiction pénale internationale et d'un code pénal international, le droit international possèdait tout de même des outils importants pour la répression des violations graves au droit humanitaire.

Le 9 décembre 1948, l'Assemblée générale adopta la Convention pour la prévention et la répression du crime de génocide. Suite à la ratification, cette convention fut mise en vigueur le 12 janvier 1951. L'article 6 de la Convention prévoit le recours à une cour criminelle internationale facultative qui sera compétente à l'égard des parties qui auront reconnu sa juridiction. Les adeptes de la compétence d'une juridiction internationale appuyaient leur argumentation sur le fait qu'en cas de génocide, « il serait impossible de se fier aux tribunaux de l'État où le crime a été commis pour qu'ils exercent leur juridiction » (AG CDI, 1949 : 36). En effet, le génocide ne peut que rarement être commis sans la participation ou la tolérance de l'État (Ternon, 1995). Leurs adversaires soutenaient qu'une juridiction internationale cons- 
tituait une atteinte à la souveraineté des États dans le domaine répressif ${ }^{12}$. Malgré de nombreux débats, cette juridiction ne fut jamais créée.

La deuxième réalisation importante a été la révision, en 1949, des quatre Conventions de Genève qui ont été ratifiées par la presque-totalité des États. Ces conventions traitent du sort des malades et blessés de la guerre, des prisonniers de guerre, des blessés, malades et naufragés des forces armées terrestres et de la protection des personnes civiles en temps de guerre. Les individus coupables des infractions contenues dans ces conventions engagent leur responsabilité pénale individuelle et peuvent être poursuivis par les juridictions nationales en vertu du système de la compétence universelle ou de la compétence territoriale.

Les États signataires se sont engagés à respecter et à faire respecter ces conventions. Cet énoncé implique que, si les États belligérants ont l'obligation de poursuivre leurs propres ressortissants auteurs de crimes de guerre, les États non belligérants ont, quant à eux, pris l'engagement de s'assurer qu'ils le fassent. De plus, dès qu'un État signataire a connaissance du fait qu'il se trouve sur son territoire une personne qui a commis un crime de guerre ou un crime contre l'humanité, il doit engager contre cette personne des poursuites ou l'extrader afin qu'elle soit jugée. Mais qu'en est-il de l'efficacité de ces mécanismes?

L'échec de la compétence universelle et de la répression nationale

Les conventions internationales existant, avant 1949, en matière de droit de la guerre, prévoyaient l'existence de sanctions en cas de violations qui n'avaient guère d'effet dissuasif étant donné leur caractère essentiellement d'ordre pécuniaire ${ }^{13}$. Les États restaient ainsi libres de poursuivre et de condamner ou non les individus coupables d'infractions graves au droit international en raison de leur souveraineté.

Afin de pallier les insuffisances d'une répression internationale l'absence d'une juridiction pénale internationale permanente - les juridictions nationales ont pris en charge la répression des infractions internationales ${ }^{14}$. La solution de la communauté internationale, afin de

12. "L'institution d'une juridiction internationale constituait une violation du droit souverain dont jouit chaque État de juger les crimes commis sur son territoire. La souveraineté des États est le fondement même de l'Organisation des Nations Unies » M. Morozov, représentant de l'U.R.S.S. (AG CDI, 1949 : 40).

13. Par exemple, voir l'article 3 de la Convention IV de La Haye du 18 octobre 1907, infra, note 18.

14. À titre d'illustration, voir Government of Israel c. Adolf Eichmann, Israel Supreme court, 29 mai 1962, 36 I.L.R. 296. 
faire respecter la nécessité de punir, tout en respectant la souveraineté des États en matière pénale, est le développement du mécanisme de la compétence universelle, appelée aussi le principe de l'universalité du droit de punir que l'on retrouve dans divers instruments internationaux. Ce principe a été retenu dans la mesure où il constituait la seule possibilité de poursuite en l'absence d'extradition.

En 1949, les Conventions de Genève ont, pour la première fois, établi un système applicable à tous les États parties : celui de la compétence universelle. Ce système devait permettre une certaine uniformisation dans la répression pénale des auteurs de violations graves du droit international humanitaire ${ }^{15}$. La répression pénale, se fondant sur le principe de la compétence universelle, comporte l'obligation de poursuivre ou d'extrader et d'accorder une entraide judiciaire en ce domaine ${ }^{16}$.

La compétence universelle, ubi te invenero, ibi te judicabo, est un mécanisme de mise en œuvre de la responsabilité pénale internationale des individus dont le but est d'assurer une répression pour les infractions graves. Elle se définit comme étant :

un système donnant vocation aux tribunaux de tout État sur le territoire duquel se trouve l'auteur de l'infraction pour connaittre de cette dernière et ce, quels que soit le lieu de perpétration de l'infraction et la nationalité de l'auteur ou de la victime (La Rosa, 1998 : 10).

Les principales conventions internationales attribuent aux États la responsabilité de faire respecter le droit international humanitaire par le biais des juridictions nationales ${ }^{17}$. Les États qui ont adhéré à ces conventions sont tenus de prendre les dispositions nécessaires afin de punir ou d'extrader les responsables de ces violations (David, 1994 : 643).

Le système de la compétence universelle exprime la solidarité des États dans la lutte contre l'impunité des violations graves aux droits de l'homme les plus fondamentaux. Ce système doit « permettre de trouver dans tous les cas un juge et réaliser ainsi par la reconnaissance de la compétence universelle de punir, l'ubiquité de la répression qui est le principe primordial du droit pénal international » (Quoc Dinh et al., 1994 : 625).

15. Le Premier Protocole additionnel de 1977 a complété les Conventions de Genève sur ce point.

16. Voir notamment l'article 88 du Premier Protocole additionnel de 1977.

17. Voir, par exemple, articles 49(I), 50(II), 129(III) et 146(IV) des Conventions de Genève; l'article 6 de la Convention pour la prévention et la répression du crime de génocide; article 85(1) du Premier Protocole additionnel de 1977; article 5 de la Convention contre la torture et autres peines ou traitements cruels, inbumains ou dégradants; articles 4 et 11 de la Convention internationale sur l'élimination et la répression du crime d'apartbeid; et article 6 de l'Accord de Londres du 8 août 1945. 
Le système de la compétence universelle a été très peu employé suite à la Seconde Guerre mondiale. Les tribunaux nationaux se révélaient inefficaces et inadéquats, soit par manque de moyens, soit par manque de volonté politique, à juger les criminels de guerre. La situation politique avait changé : c'était le début de la guerre froide. Les États ne voulaient pas limiter leur liberté de décider d'une intervention armée.

L'échec de la compétence universelle n'a malheureusement pas été compensé par une répression des tribunaux pénaux nationaux. En effet, lors de la Deuxième Guerre mondiale, devant l'étendue des crimes commis, l'Accord de Londres et la Déclaration de Moscou établissaient le principe définitif des compétences dans l'application de la répression de ces crimes. Ils définissaient la compétence d'un tribunal en fusionnant deux ordres normatifs : le droit international et les droits nationaux. Les criminels de guerre, non justiciables des tribunaux internationaux de Nuremberg et de Tokyo, devaient être jugés par les juridictions nationales. Leur compétence était déterminée par le lieu de commission des crimes. Les systèmes nationaux trouvaient, dans leur droit interne, les règles de compétence nécessaires selon le critère territorial ou la nationalité de l'auteur ou de la victime.

La répression des crimes de guerre se fit donc dans une variété de règles de compétence et de systèmes de droit. Un grand nombre de présumés coupables se trouvaient sur le territoire allemand et pouvaient être jugés par un ou plusieurs pays. Afin de répartir les compétences entre les juridictions nationales, la Loi $n^{0} 10 \mathrm{du}$ Conseil de Contrôle fut édictée. Cette loi prévoyait que son application pouvait être déléguée aux juridictions allemandes et reprenait les mêmes infractions que le Statut du Tribunal de Nuremberg.

Malgré le demi-succès des tribunaux nationaux ${ }^{18}$, de nombreux criminels de guerre, en fuite lors de la défaite allemande, échappèrent au jugement des juridictions nationales et allemandes en se réfugiant dans de nombreux pays. Encore aujourd'hui, nous retrouvons un peu partout la trace de ces criminels de guerre nazis.

18. Plusieurs milliers de condamnations furent prononcées par les tribunaux alliés et allemands. Par exemple, Rudolf Höss, commandant d'Auschwitz, fut condamné à mort, en Pologne, en 1947. Adolf Eichmann, le principal responsable de la « solution finale » fut enlevé en Argentine par un commando israélien et condamné à mort en Israël en 1961. Karl Oberg, chef suprême de la police et des SS en France, fut gracié en 1958 et libéré en 1962 par le Général de Gaulle. Klaus Barbie, chef de la Gestapo à Lyon, fut extradé en 1983 et condamné à la prison à vie en 1987. De plus, en 1958, le Service central d'enquête sur les crimes nationaux-socialistes fut créé. Jusqu'en 1964, il engagea une centaine d'enquêtes par année. 
Les infractions reconnues comme des crimes internationaux, et parmi eux les crimes de guerre, les crimes contre l'humanité et le crime de génocide, sont des actes d'une gravité exceptionnelle reconnus comme des atteintes universelles. Ces crimes internationaux ne peuvent donc pas échapper, en raison de cette gravité, aux limites d'espaces tracées par le droit interne. La répression de ces infractions constitue l'engagement de tout État par le biais de sa compétence universelle. Le principe de la souveraineté des États devrait donc céder sa place à une exigence plus élevée de la Justice, soit celle d'assurer une protection efficace et universelle des droits de l'homme les plus fondamentaux.

\section{La reconnaissance de trois crimes internationaux par la cour pénale internationale}

Le Statut de Rome reconnaît la responsabilité pénale internationale des individus pour trois catégories de crimes : les crimes de guerre, les crimes contre l'humanité et le crime de génocide.

Un apport et une déception en matière de crimes de guerre

L'expression « crimes de guerre » couvre traditionnellement les violations des règles applicables dans les conflits armés internationaux, le jus in bello, susceptibles d'engager la responsabilité pénale individuelle de leur auteur. Les violations au droit international humanitaire ne sont pas toutes des crimes de guerre; seules les violations les plus graves sont incluses : les « principales règles de fond du droit des conflits armés » (David, 1994 : 564). De tels actes avaient déjà été interdits par plusieurs instruments internationaux, notamment par les Conventions de La Haye de $1907^{19}$, qui s'étaient

19. Les Conventions de La Haye du 18 octobre 1907 mettent à jour et complètent les Déclarations de La Haye de 1899. Convention I relative au règlement pacifique des conflits internationaux, 18 octobre 1907, 54 R.T.S.N. 435; Convention IV relative aux lois et coutumes de la Guerre, 18 octobre 1907, 36 R.T.S.N. 539; Convention V relative aux droits et devoirs des Puissances et des personnes neutres en cas de guerre sur terre, 18 octobre 1907, (3 $\left.{ }^{\mathrm{e}}\right) 3$ MARTENS 504; Convention VI relative au régime des navires de commerce au début des bostilités, 18 octobre 1907, (3 $3^{\mathrm{e}} 3$ MARTENS 533; Convention VII relative à la transformation des navires de commerce en bâtiments de guerre, 18 octobre 1907, $\left(3^{\mathrm{e}}\right) 3$ MARTENS 557; Convention VIII relative à la pose des mines sous-marines automatiques de contact, 18 octobre 1907, 3 MARTENS 580; Convention IX relative au bombardement par des forces navales en temps de guerre, 18 octobre 1907, (3 $\left.3^{e}\right) 3$ MARTENS 604; Convention X relative à l'adaptation à la guerre maritime des principes de la Convention de Genève, 18 octobre 1907, 15 R.T.S.N. 340; Convention XI relative à certaines restrictions à l'exercice du droit de capture dans la guerre maritime, 18 octobre 1907, $\left(3^{e}\right) 3$ MARTENS 663; Convention XIII relative à l'établissement d'une cour internationale des prises, 18 octobre 1907, (3 $\left.3^{e}\right) 3$ MARTENS 713 et la Convention XIV relative aux droits et aux devoirs des Puissances neutres en cas de guerre maritime, 18 octobre 1907, (3 $\left.3^{e}\right) 3$ MARTENS 745. 
efforcées de réglementer les guerres en vue de les humaniser et d'éliminer les activités les plus révoltantes; cependant ces instruments n'édictaient aucune peine. Seule la responsabilité internationale de l'État pouvait être engagée pour les actes de ses forces armées (Bierzanek, 1987 : 27-50). Pour la première fois, le Statut du tribunal de Nuremberg et la Charte du tribunal de Tokyo ont incriminé ces actes en les assortissant de sanctions pénales.

Ce sont les crimes contraires aux lois et coutumes de la guerre, tels que les meurtres, tortures et mauvais traitements ${ }^{20}$, les expériences biologiques et médicales ${ }^{21}$, la prise et l'exécution d'otages ${ }^{22}$, la déportation des populations civiles ${ }^{23}$, la détention illégale des personnes civiles ${ }^{24}$, les attaques contre les personnes civiles ${ }^{25}$, l'enrôlement forcé des personnes au pouvoir de l'ennemi ${ }^{26}$, le pillage et la destruction sans motifs des biens, villes et villages ${ }^{27}$ et les atteintes aux biens culturels ${ }^{28}$.

La liste de ces violations érigées en crimes de guerre n'est pas fermée. L'article $6 \mathrm{~b}$ du Statut du tribunal de Nuremberg stipule que les violations des lois et coutumes de la guerre énumérées le sont "sans y être limitées ». Au même effet, la liste des infractions qui figurent à l'article 3 du Statut du tribunal pour l'ex-Yougoslavie est de caractère illustratif et non limitatif ${ }^{29}$.

Le Statut de Rome de la cour pénale internationale s'inscrit parfaitement dans le prolongement des expériences du passé tout en intégrant la prise en compte des besoins d'aujourd'hui. L'article $8 \mathrm{du}$ Statut de Rome peut être divisé en deux parties : la première définissant les crimes de guerre dans les conflits armés internationaux; alors que la deuxième traite des crimes de guerre dans les conflits armés non internationaux.

20. Article 6b du Statut de Nuremberg; article 5 du Statut de Tokyo; articles 50(I), 51(II), 130 (III) et 147(IV) des Conventions de Genève. Ces infractions sont aussi prévues à l'article 22(2)a) du Code des crimes contre la paix et la sécurité de l'bumanité, A/46/10, 1991 (ci-après Code des crimes).

21.Articles 50(I), 51(II), 130(III) et 147(IV) des Conventions de Genève; article 11 du Premier Protocole additionnel; article 22(2)a) du Code des Crimes.

22.Article 6b du Statut de Nuremberg; article 147 de la Convention IV de Genève; article 22(2) a) du Code des crimes.

23. Article $6 \mathrm{~b}$ du Statut de Nuremberg; articles 49 et 147 de la Convention IV de Genève; article 22(2)a) et b) du Code des crimes; article 85(4)a) du Premier Protocole additionnel.

24. Articles $147,41,42$, et 79 de la Convention IV de Genève.

25. Article 85(3) a) et b) du Premier Protocole additionnel.

26. Articles 130(III), 4(IV) et $147(\mathrm{IV})$ des Conventions de Genève; article 23 de la Convention IV de La Haye de 1907; article 22(2)a) du Code des crimes.

27. Article 6b et 6c du Statut de Nuremberg; articles 50(I), 51(II) et 147(IV) des Conventions de Genève; article 22(2)e) du Code des crimes; article 3b du Statut du Tribunal pour l'ex-Yougoslavie.

28. Article 3d) du Statut du Tribunal pour l'ex-Yougoslavie, article 27 de la Convention IV de La Haye de 1907; article 85(4)d) du Premier Protocole additionnel; article 22(2)f) du Code des crimes.

29. Le Procureur c. Martic, Tribunal pénal international pour l'ex-Yougoslavie, IT-95-13-I. 
Dans le domaine des conflits internationaux, le Statut de Rome met en forme dans un seul texte les infractions graves du droit de Genève et les violations graves des lois et coutumes de la guerre du droit de La Haye. À plusieurs occasions, le Statut développe et élargit les concepts du droit de La Haye et du Premier Protocole additionnel de 1977. Il inclut aussi quelques instruments internationaux sur la proscription de certains moyens et méthodes de combat. Il est cependant regrettable que la disposition relative à l'utilisation d'armes particulièrement cruelles a été limitée au maximum et n'inclut pas les armes nucléaires en raison de la difficulté de parvenir à un consensus des États.

Dans le domaine des conflits internes, le Statut de Rome est la consécration de la coutume en matière de droit international humanitaire (Meron, 1998 : 305). Cette codification est principalement due aux réactions de l'opinion publique mondiale face aux atrocités subies au Rwanda et en ex-Yougoslavie, à la création des deux tribunaux ad hoc et à la jurisprudence innovatrice de ces tribunaux. En effet, lors des négociations pour l'adoption du Deuxième Protocole additionnel aux Conventions de Genève, l'écrasante majorité des États avait refusé l'idée même que la notion de crime de guerre puisse être introduite dans le droit applicable aux conflits armés non internationaux.

En ce qui concerne les infractions particulières, les crimes à caractère sexuel tels que le viol, l'esclavage sexuel, la prostitution forcée, la grossesse forcée et la stérilisation forcée sont désormais qualifiés de crimes de guerre. La conscription et l'enrôlement des enfants de moins de quinze ans dans les forces armées nationales ou dans un groupe armé sont également considérés comme des crimes de guerre.

Malgré ce progrès, le Statut prévoit la possibilité pour un État d'émettre une réserve sur l'effectivité de la cour pénale internationale. En effet, tout État peut déclarer qu'il n'accepte pas la compétence de la cour en ce qui concerne les crimes de guerre pour une période de sept ans à compter de l'entrée en vigueur du Statut. (art. 124).

Une contribution en matière de crimes contre l'humanité

La notion de « crimes contre l'humanité » est apparue pour la première fois dans le Traité de Sèvres de 1920 prévoyant la responsabilité pénale des auteurs du massacre de la population arménienne, conclu entre les Alliés et le gouvernement Turc, à la fin de la Première Guerre mondiale. Ce traité ne fut jamais ratifié et le Traité de Lausanne du 24 juillet 1923 a accordé l'amnistie pour tous les crimes commis entre 1914 et 1922. 
Officiellement, le concept juridique de «crimes contre l'humanité »a été reconnu suite à la Deuxième Guerre mondiale avec l'Accord de Londres du 8 août 1945 créant le tribunal de Nuremberg (Crynfogel, 1992).

Les crimes contre l'humanité sont dirigés contre une population civile qu'ils aient ou non été commis au cours d'un conflit armé de caractère international ou interne. Il s'agit aussi de persécutions pour des motifs politique, idéologique, racial, national, ethnique ou religieux, que ces actes ou persécutions aient constitué ou non une violation du droit interne du pays où ils ont été perpétrés (Bassiouni, 1992 : 228-338; 1987 : 51-72).

La notion de crime contre l'humanité implique la reconnaissance au profit de la personne humaine de droits fondamentaux supérieurs au droit de l'État. Ces droits sont protégés, en cas de violations, par des sanctions pénales internationales. Les crimes contre l'humanité sont des crimes internationaux qui, à raison même de cette qualité, échappent aux limites du droit interne. Ils « relèvent d'un ordre répressif international auquel la notion de frontières est fondamentalement étrangère $»^{30}$. Il s'agit de l'assassinat, de l'extermination, de la réduction en esclavage, de l'expulsion, de l'emprisonnement, de la torture, du viol, des persécutions pour des raisons politiques, raciales et religieuses ainsi que des autres actes inhumains commis contre toutes populations civiles ${ }^{31}$.

Dans le droit de Nuremberg, les crimes contre l'humanité ne pouvaient être punis que pour autant qu' ils se rattachaient à des crimes de guerre ou à des crimes contre la paix (Grynfogel, 1992 : 1034; Zoller, 1993 : 549). La cour de cassation élargissait la définition du crime contre l'humanité dans l'affaire Barbie $e^{32}$. Klaus Barbie, spécialisé dans la chasse aux résistants, ne pouvait être accusé de crimes de guerre, en raison de la prescription de ses crimes, considérés comme des crimes de guerre. Le tribunal s'en tiendra à la définition de Nuremberg et déclarera que les résistants sont des combattants volontaires. Suite à cette décision, les associations de résistants décidèrent de former un pourvoi à la cour de cassation afin de faire modifier la définition du crime contre l'humanité pour que certains crimes de guerre - précisément ceux commis contre les résistants — soient

30. Cass. Crim., 6 octobre 1983, Klaus Barbie, pourvoi nº 83-94.194, (1983) Bull. crim., p. 610 .

31. Voir l'article 6c du Statut de Nuremberg; article 5c du Statut de Tokyo; article 5 du Statut du Tribunal pour l'ex-Yougoslavie; article 3 du Statut du Tribunal pour le Rwanda. Voir aussi article 2 de la Loi $n^{0} 10$ du Conseil de contrôle allié en Allemagne et article 21 du Code des crimes.

32. Cass. crim., 20 décembre 1985, Fédération nationale des déportés et mutilés résistants et patriotes et autres c. Klaus Barbie, pourvoi no 85-95.166, (1985), 407 Bull. Crim. 1038; Klaus Barbie, pourvoi n 83-94.425, (1984) Bull. Crim. 20. 
rattachés à la catégorie, élargie pour la circonstance, des crimes contre l'humanité. La cour a décidé que :

constituent des crimes contre l'humanité $[. .$.$] - alors même qu'ils se-$ raient également qualifiables de crimes de guerre selon l'article 6 (b) de ce texte - les actes inhumains et les persécutions qui, au nom d'un État pratiquant une politique d'hégémonie idéologique, ont été commis de façon systématique non seulement contre des personnes en raison de leur appartenance à une collectivité raciale ou religieuse, mais aussi contre les adversaires de cette politique, quelle que soit la forme de leur opposition.

Le Statut du Tribunal pour le Rwanda restreint les crimes contre l'humanité aux faits «commis dans le cadre d'une attaque généralisée et systématique » (art. 3) et le Statut du Tribunal pour l'ex-Yougoslavie aux faits « commis au cours d'un conflit armé, de caractère international ou interne » (art. 5). Ces limitations ratione materia s'expliquent par le caractère ad hoc de ces juridictions et se doublent de limitations ratione tempore.

La notion de " crime contre l'humanité » a beaucoup évolué depuis Nuremberg. Le Statut de Rome est le premier instrument conventionnel multilatéral à portée générale définissant de manière détaillée la liste des comportements qualifiés de crimes contre l'humanité reconnus par les instruments internationaux (art. 7). L'énumération des types d'actes représentant ces crimes reprend celle du Statut de Nuremberg (art. 6) avec des ajouts et celles des Statuts du tribunal pour l'ex-Yougoslavie (art. 5) et du tribunal pour le Rwanda (art. 3). Comme pour les crimes de guerre, le Statut de Rome élargit la notion de crimes contre l'humanité en y ajoutant les actes à caractère sexuel.

Contrairement au droit de Nuremberg, le Statut de Rome prévoit que le crime contre l'humanité peut être commis en dehors d'un conflit armé tant international qu'interne. Il peut donc s'appliquer à toutes les violations graves des droits de l'homme dès qu'elle se rattache à une politique systématique et ce, même en temps de paix. La communauté internationale a désormais à sa disposition un texte cohérent en matière de crimes contre l'humanité qui vise à remédier à la dispersion. Ce texte, tout en étant le prolongement de ce qui a déjà été fait, intègre les besoins d'aujourd'hui.

Le statu quo en matière de génocide

En 1946, la notion de « génocide » apparaît pour la première fois dans une résolution de l'Assemblée générale des Nations Unies. Elle définit le crime de génocide comme un «crime du droit des gens, condamné par le monde civilisé » et comme étant un crime qui «bouleverse la cons- 
cience humaine, inflige de grandes pertes à l'humanité et est contraire à la loi morale ainsi qu'à l'esprit et aux fins des Nations Unies » ${ }^{33}$. En 1948, cette condamnation sera confirmée par la Convention pour la prévention et la répression du crime de génocide adoptée par l'Assemblée générale à l'unanimité ${ }^{34}$.

Le crime de génocide peut être commis en temps de paix ou en temps de guerre (art. 1). Selon la définition de l'article 2 de la Convention, le crime de génocide se caractérise par trois éléments constitutifs.

Le premier élément est matériel. Il comprend l'énumération de l'article 2 de la Convention, et exclut les dommages causés aux biens et le génocide culturel (destruction de la langue, de la religion ou de la culture d'un groupe); seules les atteintes aux personnes sont prises en considération.

Le deuxième élément est moral. C'est l'intention coupable requise, car il ne peut y avoir de génocide sans « l'intention de détruire, en tout ou en partie, un groupe ». Cette intention constitue le trait caractéristique du génocide par rapport au crime contre l'humanité ou au meurtre : «Ce n'est pas le mode d'agir mais le but de la destruction qui constitue l'élément distinctif du crime de génocide » (Plawski, $1972: 115)$.

La distinction entre génocide et crime contre l'humanité n'est pas toujours parfaitement claire. Contrairement à l'auteur d'un crime contre l'humanité, l'auteur d'un génocide cherche à atteindre un groupe. Certains auteurs ont pour cette raison considéré le génocide comme étant « un cas aggravé ou qualifié de crime contre l'humanité » étant donné l'intention renforcée qui caractérise le génocide (Glaser, 1954 : 4-5; Glaser, 1970 : 120; Ternon, 1995 : 46). La différence est d'ordre subjectif et porte sur l'intention:

Si le criminel agit en vue de supprimer sa victime en raison de sa race, de sa religion ou de ses convictions politiques, sans autre intention, c'est un crime contre l'humanité. S'il a l'intention de détruire un groupe national, ethnique, racial ou religieux, en tout ou en partie, c'est un génocide. La différence réside essentiellement dans une particularité de l'intention criminelle (Plawski, 1972 : 9-10).

Et le troisième élément est le fait que le génocide requiert que l'acte criminel vise un groupe, qu' il soit national, ethnique, racial ou religieux.

33. Résolution 96(1), Assemblée générale, Nations Unies, 11 décembre 1946. La proposition a été rédigée par le créateur du terme " génocide » : Raphaël Lemkin (1944 : 79$)$.

34. La définition du crime de génocide sera aussi reprise par l'article 19 du Code des crimes. 
La Convention ne s'applique pas aux actes visant à détruire un groupe politique, idéologique, économique ou social ${ }^{35}$.

L'article 4 du Statut du Tribunal pour l'ex-Yougoslavie, l'article 2 du Statut du Tribunal pour le Rwanda et l'article 6 du Statut de Rome reprennent intégralement la définition du génocide de la Convention ${ }^{36}$.

La Convention pour la prévention et la répression du crime de génocide n'a pratiquemment jamais été appliquée, parce que les États contractants n'ont jamais pris les mesures d'exécution requises, au niveau de la prévention ou au niveau de la répression, à la mise en œuvre effective de ces dispositions. La répression du génocide ne sera vraiment effective que si elle est internationale puisque le génocide est un crime directement lié à l'État (Ternon, 1995 : 46). Le crime de génocide ne peut être commis sans la complicité de l'État et, par le fait même, ne peut être réprimé par ce même État.

\section{Les compromis de la communauté internationale}

Les mécanismes de mise en œuvre du droit international existant ont échoué au niveau du jugement des criminels de guerre et de la lutte contre l'impunité. Afin de remédier à cet échec, la communauté internationale a établi une justice pénale internationale par divers compromis. D'une part, elle a multiplié, pour des besoins spécifiques, la création de tribunaux pénaux ad hoc, dont il a fallu justifier la création et limiter les compétences, et, d'autre part, elle a créé une juridiction pénale internationale permanente ayant une compétence affaiblie et soumise et dont la complémentarité avec les juridictions nationales ressemble à une subsidiarité.

\section{La multiplication des tribunaux pénaux internationaux ad hoc}

Les horreurs de la Deuxième Guerre mondiale que l'on croyait révolues, ou réservées à des régions lointaines, les guerres civiles et internationales où de nombreux massacres ont lieu se déroulent maintenant en Europe.

35. L'exclusion concernant les groupes politiques trouve son explication dans la « seule volonté politique d'une majorité de voir une convention sur la prévention et la répression du crime de génocide ratifiée par les États socialistes qui l'eussent catégoriquement rejetée si les groupes politiques avaient été visés dans la définition de celui-ci » (Verhœven, 1991 : 21).

36. La pratique contemporaine révèle cependant une extension de la notion de génocide à la notion de " nettoyage ethnique », c'est-à-dire un large spectre de violations grossières et systématiques des droits de l'homme. Résolution 47/121, Assemblée générale, Nations Unies, 18 décembre 1992. 
La bonne conscience de la communauté internationale ne peut se satisfaire d'une condamnation symbolique par les organes des Nations Unies comme cela se passe généralement pour les guerres se déroulant en Asie, en Afrique ou en Amérique Latine. La Yougoslavie est un pays européen et la situation politique de l'Europe n'est plus la même. Elle n'est plus divisée en deux blocs opposés : la guerre froide est terminée.

Trois membres permanents du Conseil de sécurité sont Européens et un quatrième, les États-Unis, sont des alliés stratégiques au sein de l'O.T.A.N. De plus, le conflit interne pourrait avoir des conséquences, telles qu'amener un flot de réfugiés sur les zones frontalières des pays voisins. La communauté internationale a créé le tribunal pénal international pour l'ex-Yougoslavie afin de préserver la paix sur le territoire européen. Suite aux pressions internationales et au précédent qu'elle venait de créer, elle a dû établir un autre tribunal international ad boc, mais cette fois-ci pour l'Afrique : le tribunal international pour le Rwanda.

La création de deux juridictions pénales internationales ad boc - le tribunal pénal international pour l'ex-Yougoslavie et le tribunal pénal pour le Rwanda - sont une grande " deuxième " après les tribunaux de Nuremberg et de Tokyo. Cette création constitue une étape pour briser le cycle d'impunité face aux violations flagrantes du droit international humanitaire. Les États ont depuis longtemps l'obligation de réprimer les comportements constituant des crimes de guerre, des crimes contre l'humanité et des crimes de génocide par le biais de la compétence universelle. Par le fait même de leur création, les deux tribunaux sont l'aveu de l'échec du respect de cette obligation que les États ont contractée.

Ces tribunaux, contrairement à Nuremberg et à Tokyo, ne représentent pas la justice des vainqueurs, mais est-ce une justice désintéressée ? Ne sont-ils pas qu'un geste politique symbolique destiné à satisfaire à court terme les intérêts politiques des États ? Les États, ne se sentant pas prêts à assumer individuellement leur rôle quant à la justice pénale internationale, soit de « respecter et faire respecter » le droit international humanitaire, ont créé une justice $a d b o c^{37}$.

37. «Les parquets d'un État sont beaucoup plus enclins à poursuivre l'auteur d'un détournement d'avion commis aux antipodes qu'à poursuivre un tortionnaire ou un chef de guerre qui, dans un État voisin a, avec l'accord de son gouvernement, participé à la torture ou au massacre de dizaines, de centaines ou de milliers de personnes !» (David, 1992 : 597). 
La justification de leur création

Le tribunal pénal international ad hoc offre le mérite de répondre à un besoin spécifique, défini avec précision, et de punir les crimes commis. À la différence des tribunaux de Nuremberg et de Tokyo, les tribunaux pour l'ex-Yougoslavie et pour le Rwanda ne sont pas là pour appliquer le droit des vainqueurs à des ennemis vaincus, mais pour sanctionner les coupables de violations graves du droit humanitaire au nom de la communauté internationale tout entière sans faire de distinctions selon l'idéologie des accusés ou le camp dans lequel ils se sont rangés. De plus, un tribunal ad hoc a l'avantage de pouvoir être créé rapidement.

Deux tribunaux furent créés par le Conseil de sécurité, en vertu du chapitre VII de la Charte des Nations Unies, afin de mettre fin aux violations graves du droit humanitaire et aux actes de génocide commis en ex-Yougoslavie et au Rwanda. Le Conseil de sécurité décide, le 22 février 1993, « la création d'un tribunal international pour juger les personnes présumées responsables de violations graves du droit humanitaire international commises sur le territoire de l'ex-Yougoslavie depuis $1991 »^{38}$ et, le 8 novembre 1994, « de créer un tribunal international chargé uniquement de juger les personnes présumées responsables d'actes de génocide ou d'autres violations graves du droit international humanitaire commis sur le territoire du Rwanda et les citoyens rwandais présumés responsables de tels actes ou violations commis sur le territoire d'États voisins, entre le $1^{\text {er }}$ janvier et le 31 décembre $1994 »^{39}$.

En effet, la création de ces tribunaux par voie de traité a été écartée pour des raisons d'ordre pratique comme l'indique le Secrétaire général ${ }^{40}$. Par le biais des articles 24, alinéa 1, 39, 41 et 42 du Chapitre VII de la Charte des Nations Unies, traitant de la menace contre la paix et la sécurité internationales, le Conseil de sécurité a pu créer le tribunal pénal international pour l'ex-Yougoslavie afin de rétablir la paix. Pour respecter les exigences du Chapitre VII (Cot et Pellet, 1985 : 39-42; Lescure, 1994 : 77-85), le Conseil de sécurité a qualifié, dès le départ, la crise yougoslave

38. Résolution 808, Conseil de sécurité, Nations Unies, 22 février 1993.

39. Résolution 955, Conseil de sécurité, Nations Unies, 8 novembre 1994.

40. «Comme il est indiqué dans beaucoup des observations reçues, la méthode conventionnelle présente cet inconvénient qu'il faut beaucoup de temps pour établir un instrument et pour obtenir le nombre de ratifications requis pour son entrée en vigueur » : Rapport du Secrétaire général établi en application du paragraphe 2 de la Résolution 808 (1993) du Conseil de sécurité, op. cit. note 2, para. 20, p. 8. 
de «menace contre la paix et la sécurité internationales $»^{41}$. Conformément au précédent, le tribunal pour le Rwanda fut institué au terme de ce même chapitre.

Seul le Chapitre VII justifiait l'intervention du Conseil de sécurité dans ce domaine. L'article 41 de la Charte des Nations Unies autorise expressément le Conseil de sécurité de décider de «quelles mesures n'impliquant pas l'emploi de la force armée doivent être prises pour donner effet à ses décisions ». L'article 29, quant à lui, permet au Conseil de sécurité « de créer les organes subsidiaires qu'il juge nécessaires à l'exercice de ses fonctions ».

La création d'une juridiction pénale en tant qu'organe subsidiaire du Conseil de sécurité peut relever de sa compétence dans la mesure où il lui parait de nature à donner effet à ses décisions. Le recours aux mesures envisagées par l'article 41 doivent cependant réunir les conditions préalables, à savoir procéder à la qualification de la situation en regard de l'article 39 (Cot et Pellet, 1985 : 691-704). Le Conseil de sécurité a maintenu cette ligne de conduite ${ }^{42}$ et a, par le fait même, procédé à la qualification exigée par l'article 39 (Petrovic et Condorelli, 1992). Le préalable nécessaire à l'adoption des mesures de l'article 41 était donc rempli. Le Conseil de sécurité s'était ainsi placé dans le cadre du Chapitre VII. Il a préparé, par le moyen d'une longue série de résolutions, la création d'une juridiction pénale $a d b o c^{43}$.

Étant donné que le tribunal pour l'ex-Yougoslavie a été créé par une décision du Conseil de sécurité, son statut et les conséquences juridiques qui en découlent s'imposent à tous les États ${ }^{44}$. Adoptant le même

41. Voir Résolution 713, Conseil de sécurité, Nations Unies, 25 septembre 1991; Résolution 752, Conseil de sécurité, Nations Unies, 15 mai 1992; Résolution 764, Conseil de sécurité, Nations Unies, 13 juillet 1992; Résolution 771, Conseil de sécurité, Nations Unies, 13 août 1992; Résolution 798, Conseil de sécurité, Nations Unies, 18 décembre 1992; Résolution 808, Conseil de sécurité, Nations Unies, 22 février 1993.

42. Idem.

43. Ces résolutions visaient à respecter le droit international humanitaire, à constater que la situation constituait une «menace pour la paix et la sécurité internationales », et à affirmer la responsabilité pénale individuelle des individus responsables des violations au droit international humanitaire. (Idem).

44. Article 24 de la Charte des Nations Unies. Le pouvoir du Conseil de sécurité de créer une juridiction pénale internationale dans le cadre de son mandat de protection de la paix et de la sécurité internationales, à savoir en vertu du Chapitre VII, a été confirmé par la chambre d'appel du tribunal international pour l'ex-Yougoslavie (Le Procureur c. Tadic, 2 octobre 1995, IT-94-1-AR72.) 
procédé $^{45}$, le Conseil de sécurité a créé, par sa résolution 955, le tribunal international pour le Rwanda.

Étant des organes subsidiaires du Conseil de sécurité, les juridictions pénales ad hoc sont soumises à celui-ci. L'existence de ces tribunaux est liée à la restauration et au maintien de la paix et de la sécurité internationales. À partir du moment où le Conseil de sécurité constatera que leur existence ne sert plus les objectifs de paix et de sécurité internationales, il pourra mettre fin à leur existence.

La limitation des compétences

Ces juridictions pénales ad hoc, en leur qualité d'organes subsidiaires du Conseil de sécurité, voient leurs compétences limitées. Elles ont été créées pour des fins déterminées. Elles ne pouvaient donc être dotées que des compétences strictement nécessaires à l'atteinte de l'objectif de leur création, soit la cessation des violations du droit international humanitaire constituant une « menace à la paix et à la sécurité internationales ».

En ce qui concerne la compétence ratione loci, le tribunal pour l'exYougoslavie ne peut sanctionner que les crimes commis sur le « territoire de l'ancienne République fédérative socialiste de Yougoslavie » (art. 8), y compris son espace terrestre, son espace aérien et ses eaux territoriales. Le tribunal pour le Rwanda a compétence sur le territoire du Rwanda et celle-ci s'étend aux « territoires d'États voisins » (art. 7).

En ce qui concerne la compétence ratione tempore, le tribunal pour l'ex-Yougoslavie n'est compétent qu'à l'égard des crimes commis à partir du $1^{\text {er }}$ janvier 1991 (art. 8), date indiquant, selon le Conseil de sécurité, le début des hostilités sur le territoire yougoslave. Le tribunal pour le Rwanda reconnaît les violations commises entre le $1^{\text {er }}$ janvier 1994 et le 31 décembre 1994; ce qui le distingue de celui de l'ex-Yougoslavie dont aucune date limitant sa juridiction n'a été établie (art. 7).

Dans le cas du tribunal pour le Rwanda, même si cette solution a été adoptée afin que la planification du génocide et des crimes contre l'humanité n'y échappe pas, il n'en reste pas moins qu'elle comporte des limites : les crimes commis au Rwanda en 1994 n'étaient que le point culminant d'un long processus (Human Rights Watch et al., 1999). Ce

45. Résolution 918, Conseil de sécurité, Nations Unies, 17 mai 1994; Résolution 925, Conseil de sécurité, Nations Unies, 8 juin 1994; Résolution 929, Conseil de sécurité, Nations Unies, 22 juin 1994. Ces résolutions ont constaté l'existence d'une «menace contre la paix et la sécurité internationales » dans la région des Grands Lacs. 
paradoxe est une des principales critiques à l'encontre du tribunal pour le Rwanda car le Statut ne considère pas les causes et la planification du génocide ${ }^{46}$.

Cette compétence d'attribution limitée dans l'espace et dans le temps est due au fondement juridique, soit le Chapitre VII de la Charte, de la création de ces tribunaux. Le problème réside dans le fait qu'il ne pouvait être question pour ces tribunaux de juger tous les crimes internationaux, fussent-ils imprescriptibles, commis sur les territoires Yougoslave et Rwandais depuis l'existence de ces pays. Cela aurait été incompatible avec leur mode de création, car le Conseil de sécurité ne peut créer une juridiction que dans l'objectif de rétablir la paix et la sécurité internationales et non pas pour sanctionner des crimes anciens.

La compétence ratione personae des deux tribunaux ad hoc est limitée aux personnes physiques, soit les auteurs, co-auteurs, complices et instigateurs des crimes prévus par le Statut ${ }^{47}$. Contrairement à Nuremberg, ils n'ont pas compétence pour poursuivre pénalement les personnes morales ou privées et ne peuvent déclarer criminels des groupes, des associations ou des organisations ${ }^{48}$.

La réussite de ces tribunaux dépendra en grande partie de la volonté des États à régler les obstacles de leur mise en marche et de leur efficacité. En effet, tout le système de ces tribunaux est fondé sur la coopération des États. Si les États ne font pas preuve de bonne volonté, ces tribunaux pourraient n'être que la manifestation de la bonne conscience de la communauté internationale et devenir des «tribunaux-alibis ${ }^{49}$.

46. La guerre civile débuta au Rwanda en 1990. Le génocide d'avril 1994 fut le résultat d'une longue période de planification. C'est, entre autres, pour cette raison que le gouvernement rwandais, après avoir demandé la création d'un tribunal international, vota contre la Résolution 955 du Conseil de sécurité. Voir Conseil de sécurité, Déclaration de M. Bakuramtsa au nom du Rwanda, Explications de vote concernant la résolution 955 (1994) créant le tribunal ad hoc pour le Rwanda, 8 novembre 1994, Doc. S/PV.3453.

47. Article $6 \mathrm{du}$ Statut du Tribunal pour l'ex-Yougoslavie et art. 5 du Statut du Tribunal pour le Rwanda.

48. «La question se pose toutefois de savoir si une personne morale, telle une association, peut être considérée en tant que telle, comme auteur d'un crime, ses membres étant alors, pour cette seule raison, soumis à la juridiction du Tribunal international. Le Secrétaire général pense que ce concept ne devrait pas être retenu en ce qui concerne le Tribunal international. Les actes criminels énoncés dans les statuts sont exécutés par des personnes physiques; ces personnes seraient soumises à la juridiction du Tribunal international, indépendamment de leur appartenance à des groupes », Conseil de sécurité, Rapport du secrétaire général établi en application du paragraphe 2 de la Résolution 808 (1993) du Conseil de sécurité, op. cit., note 2, p. 165.

49. L'expression est d'Alain Pellet. 
La multiplication des tribunaux ad hoc est le compromis de la communauté internationale afin de redonner une certaine crédibilité à l'Organisation des Nations Unies.

Les événements de l'ex-Yougoslavie et du Rwanda ont permis d'accomplir un progrès remarquable en matière de responsabilité pénale internationale de l'individu. Le respect du « sacro-saint » principe de la souveraineté des États en droit international public a subi quelques brèches : l'approche est de plus en plus fondée sur la protection de la personne et sur le respect des droit de l'homme les plus fondamentaux. Ces événements ont aussi permis de reprendre les négociations et d'adopter le Statut de Rome créant la cour pénale internationale permanente.

\section{La création de la cour pénale} internationale permanente

La question de la création d'une cour pénale internationale permanente est à l'étude depuis 1920 afin de juger les individus accusés de crime de guerre, de crime contre l'humanité ou de crime de génocide. Le droit international pose des règles de droit pénal mais en laisse l'application aux États parties aux diverses conventions. La mise en œuvre de la répression pénale internationale doit tenir compte de la souveraineté des États et de leur compétence répressive.

La fin de la guerre froide a ramené à l'ordre du jour le débat sur la création d'une cour pénale internationale permanente. Le 25 novembre 1992, l'Assemblée générale des Nations Unies prie la Commission du droit international (CDI) d'entreprendre l'élaboration d'un projet de statut d'un tribunal pénal international ${ }^{50}$. Ayant terminé ses travaux, la CDI dépose, le $1^{\text {er }}$ septembre 1994, à l'Assemblée générale des Nations Unies son rapport sur le Projet de statut d'une cour criminelle internationale (Assemblée générale, Commission du droit international, 1994). Accueillant favorablement le rapport et les recommandations de la CDI, l'Assemblée générale décide de créer, le 9 décembre 1994, un comité ad hoc « chargé d'examiner les principales questions de fond et d'ordre administratif que soulève le projet de statut préparé par la Commission [...] et d'envisager les dispositions à prendre $»^{51}$.

50. Résolution 47/33, Assemblée générale, Nations Unies, 25 novembre 1992. Voir aussi Résolution 48/31, Assemblée générale, Nations Unies, 9 décembre 1993, demandant à la CDI d'élaborer à titre prioritaire un projet de statut.

51. Résolution 49/53, Assemblée générale, Nations Unies, 9 décembre 1994. 
Suite aux réunions du comité ad hoc, l'Assemblée générale des Nations Unies a créé un comité préparatoire pour examiner plus à fond les mêmes questions et élaborer un texte de synthèse largement acceptable pour une convention ${ }^{52}$. Le comité préparatoire a achevé ses travaux le 3 avril 1998 et a soumis un projet de convention portant la création d'une cour criminelle internationale permanente. La Conférence diplomatique de plénipotentiaires des Nations Unies sur la création d'une cour criminelle internationale s'est réunie à Rome du 15 juin au 17 juillet 1998 afin d'examiner le projet et d'adopter le Statut de la cour pénale internationale ${ }^{53}$.

Après des années d'efforts et cinq semaines de négociations intenses et ardues, le Statut de la Cour pénale internationale a été adopté à Rome le 17 juillet $1998^{54}$. L'ultime but était atteint : la création de la première juridiction pénale internationale à caractère permanent et à vocation universelle. La Convention est maintenant ouverte à la signature et à la ratification des États. L'adoption de ce traité attendu depuis longtemps marque un progrès important dans la lutte contre l'impunité. Elle instaure un ordre juridique international permettant de traduire en justice ceux qui ont commis les crimes de portée internationale les plus odieux et de contribuer à la lutte contre l'impunité.

Ce pas, qualifié d'historique par tous, constitue une étape importante vers un respect plus grand du droit international humanitaire et des droits de l'homme les plus fondamentaux. Les mécanismes de mise en œuvre de la répression des crimes internationaux comportent des insuffisances évidentes. La cour pénale internationale ne pourra mettre un terme aux atrocités, mais elle pourra cependant avoir un effet dissuasif. Par contre, le Statut est un texte de compromis et il contient d'énormes lacunes.

Une compétence affaiblie et soumise

Pour faciliter l'adhésion au traité, la cour pénale internationale n'exercera sa juridiction qu'à l'égard des crimes commis postérieurement à l'entrée en vigueur de son Statut (art. 11). Le domaine de compétence ratione materiae de la cour est directement relié au maintien de la paix et de la sécurité internationales. La compétence est minimale et restreinte

52. Résolution 50/46, Assemblée générale, Nations Unies, 11 décembre 1995.

53. Résolution 52/160, Assemblée générale, Nations Unies, 15 décembre 1997.

54. Il faut souligner ici le rôle important joué par M. Philippe Kirsch, représentant canadien, qui a présidé les négociations de Rome et qui préside actuellement le comité sur la mise en œuvre. 
à un noyau dur d'infractions internationales, c'est-à-dire aux violations graves et massives des droits de l'homme : les crimes de guerre, les crimes contre l'humanité, le génocide et l'agression. La compétence de la cour est suspendue en ce qui concerne l'agression jusqu'à ce que les États s'entendent sur la définition de ce crime.

La cour peut être saisie par trois modes différents (art. 13). Les États parties au Statut de Rome peuvent saisir la cour, mais ils sont rarement en mesure d'identifier les responsables impliqués dans des violations graves des droits de l'homme. Le procureur peut ouvrir une enquête de sa propre initiative s'il y est autorisé par la chambre préliminaire de la cour (art. 15). Ce contrôle a été mis sur pied parce que les États n'ont pas voulu reproduire l'auto-saisine des deux tribunaux pénaux internationaux ad hoc afin d'éviter que le procureur utilise sa compétence et son action à des fins plus politiques.

Le Conseil de sécurité se voit reconnaître un droit de saisine fondé sur le Chapitre VII (paix et sécurité internationales) et ce, sans l'obligation de coopération générale demandée aux États parties. Qu'arrivera-t-il lorsque certains membres du Conseil de sécurité décideront de s'opposer à la saisine ? Certaines difficultés sont à prévoir étant donné que deux membres importants du Conseil de sécurité (les États-Unis et la Chine) refusent d'adhérer au traité et, par le fait même, de reconnaitre la cour pénale internationale.

Le Statut de Rome, tentant de concilier le maintien de la paix et l'action judiciaire, prévoit aussi que le Conseil peut suspendre les enquêtes et les poursuites par une demande basée sur le Chapitre VII pour une durée de douze mois, renouvelables (art. 16). Le problème réside dans le fait que l'article 103 de la Charte des Nations Unies affirme que les obligations du Conseil prévalent sur tout autre engagement international. Le Conseil peut donc imposer aux États membres des Nations Unies une interruption plus longue que douze mois de leur coopération avec la cour, et cette décision prévaudra sur les dispositions du Statut de Rome. En pratique, le Statut mise sur la bonne volonté du Conseil de sécurité (Weckel, 1998 : 991; Sur, 1999 : 44-45).

Une juridiction complémentaire ou subsidiaire ?

Le principe de complémentarité entre la cour pénale internationale et les juridictions pénales nationales est consacré dès le Préambule (par. 10) et réaffirmé à l'article premier du Statut de Rome. Dans un même souffle, les rédacteurs du Statut affirment qu'il « est le devoir de chaque État de 
soumettre à sa juridiction criminelle les responsables de crimes internationaux » (par. 6). La cour ne pourra pas intervenir si une juridiction interne exerce sa compétence (art. 17). Elle devient un mécanisme de soutien aux juridictions nationales en difficulté, car elle ne peut intervenir que dans les cas de mauvaise volonté d'un État ou dans les cas d'incapacité en raison de l'effondrement ou de l'indisponibilité de l'appareil judiciaire.

La cour pénale internationale a besoin de la coopération des États pour fonctionner et ce, particulièrement en matière d'arrestation, de remise des personnes poursuivies, d'obtention d'informations et de témoignages (art. 86 à 102). L'obligation générale de coopération (art. 86) doit être incluse dans les législations nationales (art. 88). Il risque d'être difficile de faire coopérer un État ayant été déclaré incompétent, incapable ou de mauvaise foi par la cour. Par exemple, les États visés pourront tenter de mettre en échec la justice internationale en créant leur propres procédures servant à exonérer leurs ressortissants menacés de poursuites devant la cour (Sur, 1999 : 42-43).

Le Statut prévoit que le défaut de coopération des États sera référé par la cour à l'Assemblée des États parties (art. 87(7) et 112(2)(f)). Le Statut est par contre silencieux sur les mesures qui peuvent être prises pour un refus de coopération. De plus, ces mesures ne semblent pas obligatoires pour les États parties.

Étant donné que les compétences étatiques sont le droit commun et que la cour a besoin de la coopération des États pour son fonctionnement, il semble qu'elle devrait être qualifiée de subsidiaire et non de complémentaire (Sur, 1999 : 42).

\section{Conclusion}

La multiplication des conflits intra-étatiques et l'impunité dont bénéficie les personnes responsables des crimes de guerre, des crimes contre l'humanité et des crimes de génocide ont obligé les Nations Unies à élargir son champ d'action pour sauvegarder « la paix et la sécurité internationales ». Les normes et les pratiques du peacekeeping traditionnel ne s'appliquent presque plus. Pour faire face au caractère endémique de ces conflits, l'Organisation des Nations Unies est passée d'un statut de gardien de la paix à celui d'artisan de la paix.

Le principe consacré de la non-intervention dans les affaires intérieures d'un État ne fait plus obstacle à l'action internationale (Bettati, 1996). Le concept de la souveraineté cède peu à peu sa place à des prin- 
cipes de sauvegarde et de préservation de la vie humaine et à l'émergence d'un droit d'ingérence. Les missions de paix ont des tâches de plus en plus diversifiées : maintien de l'ordre public, aide humanitaire, désarmement, surveillance des élections, reconstruction des institutions, justice répressive, démocratie, etc.

L'action des missions militaires internationales a montré ses limites à l'égard des missions de paix. L'affaiblissement du principe de la souveraineté des États dans le cadre de la sauvegarde de la paix et la sécurité internationales a fait en sorte que plusieurs missions ont pu être déployées sans le consentement des États belligérants. L'ouverture de cette brèche a été fortement dénoncée par les États et a amené la question de la sous-traitance de l'exécution des missions aux organisations régionales telles que l'O.T.A.N. Il n'en reste pas moins que la reconnaissance du droit d'ingérence par les États reste improbable et comporte des risques élevées au niveau diplomatique et politique.

La création de la cour pénale internationale est le constat de l'échec des missions de maintien de la paix. D'une part, les mécanismes de prévention mis en place dans le cadre des Nations Unies ne fonctionnent pas et, d'autre part, il semble impossible de corriger leur défaillance. Par cette création, la communauté internationale tente de réparer les conséquences de leur défaillance; c'est l'institutionnalisation de cet échec (Sur, 1999).

La cour pénale internationale ne sera efficace que si sa compétence est universelle; il ne suffit pas que justice soit juste, il faut aussi qu'elle le paraisse. Malgré le vote positif de 140 États, l'universalité de la compétence de la cour pénale internationale a été manquée. En effet, le vote négatif de sept États en plus du vote des États qui se sont abstenus est important, non en terme de vote, mais en terme de diversité et d'importance. En effet, deux membres importants du Conseil de sécurité, les États-Unis et la Chine, se sont opposés. De plus, l'Inde et Israël sont aussi parmi les opposants et les pays abstentionnistes sont majoritairement arabes. Le refus américain pourrait politiquement priver le Statut de Rome de son entrée en vigueur. Tous ensemble, ces États représentent la "moitié de l'humanité »! « Le soutien étatique massif ne doit pas faire illusion» (Sur, $1999: 38-42)$.

La judiciarisation internationale des criminels de guerre, responsables des violations graves du droit international humanitaire, par le biais d'une cour pénale internationale, est une solution beaucoup plus viable politiquement que l'acceptation d'un droit d'ingérence par les États. Elle est aussi une solution réclamée tant par l'opinion publique mondiale que 
par les victimes de ces crimes. Le maintien de la paix et la sécurité internationale passent nécessairement par la lutte contre l'impunité des personnes responsables des violations graves du droit international humanitaire, car l'impunité constitue souvent une partie intégrante des conflits intra-étatiques actuels.

Il reste néanmoins à espérer que cette nouvelle cour verra le jour dans un avenir pas si lointain ${ }^{55}$. Malgré les lacunes et les limites qui guettent son effectivité, elle contribuera à améliorer de façon significative le respect du droit international humanitaire d'une façon préventive. L'établissement de règles de preuve et de procédure du droit pénal international influencera, à moyen terme, les systèmes de droit pénal nationaux et fera avancer la démocratie et humaniser la répression criminelle dans les États. La jurisprudence de cette juridiction pénale internationale permanente inspirera les tribunaux nationaux à respecter les règles de justice considérées comme étant fondamentales à leur fonctionnement.

\section{Références}

Accord de Londres, 8 août 1945, 82 R.T.N.U. 279.

Assemblée générale, Commission du droit international (1949). Historique du problème de la juridiction criminelle internationale, Mémorandum du Secrétaire général, Lake Success, New-York, A/CN.4/7/Rev.1.

Assemblée générale, Commission du droit international (1994). Rapport de la Commission du droit international sur les travaux de sa quarante-sixième session sur le Projet de statut d'une cour criminelle internationale, A/49/355.

BASSIOUNI, M.C. 1987. "Crimes against Humanity", Pp. 51-72 in International Criminal Law: Enforcement, sous la direction de M.C. Bassiouni. New York : Transnational Publishers inc.

BASSIOUNI, M.C. 1992. Crimes against Humanity in International Criminal Law, Dordrecht : Nijhoff.

BetTATI, M. 1996. Le droit d'ingérence: mutation de l'ordre international, Paris : O. Jacob.

BIERZANEK, R. 1987. "War Crimes: History and Definition", Pp. 27-50 in International Criminal Law Enforcement, sous la direction de M.C. Bassiouni. New York : Transnational Publishers inc.

Charte des Nations Unies, 26 juin 1945, R.T. Can., no 7.

55. Au 21 décembre 1999, le Statut de Rome avait récolté 92 signatures et 6 ratifications, le tout sur les 160 délégations présentes lors de la Conférence diplomatique de plénipotentiaires des Nations Unies sur la création d'une cour criminelle internationale. 60 ratifications sont nécessaires pour la création de la cour pénale internationale et l'entrée en vigueur du Statut. 
COT, J.-P. et PELLET, A. 1985. La Charte des Nations Unies - Commentaires article par article, Paris : Economica, Bruxelles : Bruylant.

CRYNFOGEL, C. 1992. "Un concept juridique en quête d'identité : le crime contre l'humanité » Revue générale de droit international public 63 : 1027-1048.

DAVID, E. 1992. «Le tribunal international pour l'ex-Yougoslavie » Revue belge de droit international 2 : 565-598.

David, E. 1994. Principes de droit des conflits armés, Bruxelles : Bruylant.

Déclaration du Commandant suprême des Forces alliées en Extrême-Orient, 19 janvier 1946, Mémorandum du Secrétaire général, A/CN.4/5, 1949.

GLASER, S. 1954. Introduction à l'étude du droit international pénal, Paris : Librairie du recueil Sirey.

GLASER, S. 1970. Droit international pénal conventionnel, Bruxelles : Bruylant.

Human Rights Watch et Fédération internationale des ligues des droits de l'homme 1999. Aucun témoin ne doit survivre. Le génocide au Rwanda, Paris: Karthala.

LA Rosa, A.-M. 1998. Dictionnaire de droit international pénal, Paris: Presses Universitaires de France.

LEMKIN, R. 1944. Axis Rule in Occupied Europe, Washigton: Carnegie Endowment for International Peace.

LESCURE, K. 1994. Le tribunal pénal international pour l'ex-Yougoslavie, Paris : Montchrestien.

Loi no 10, Conseil de contrôle allié en Allemagne, 1945. 15 L.R.T.W.C. 41. Voir aussi l'article $21 \mathrm{du}$ Code des crimes contre la paix et la sécurité de l'bumanité.

Lombois, C. 1979. Droit pénal international, Paris : Dalloz.

Meron,T. 1998. War Crimes Comes of Age, Oxford.

PELLA, V.V. 1964. La guerre-crime et les criminels de guerre. Réflexions sur la justice internationale, ce qu'elle est et ce qu'elle devrait être, Neuchatel : Éditions de la Baconnière.

Pellet, A. 1994. "Le tribunal criminel international pour l'ex-Yougoslavie. Poudre aux yeux ou avancée décisive ? » Revue générale de droit international public 78 (1) : 7-60.

Petrovic, D. et Condorelli, L. 1992. «L'ONU et la crise yougoslave » Annuaire français de droit international 38 : 32-60.

PICTET, J. 1986. «Le droit international humanitaire : définition », Pp. 13-16 in Les dimensions internationales du droit bumanitaire, Institut Henry Dunant. UNESCO : Pédone.

PlaWsKI, S. 1972. Étude des principes fondamentaux du droit international pénal, Paris : Librairie générale de droit et de jurisprudence.

Quoc Dinh, N., Daillier, P. et Pellet, A. 1994. Droit international public, Paris : L.G.D.J.

SUR, S. 1999. "Vers une cour pénale internationale : la Convention de Rome entre les ONG et le Conseil de sécurité » Revue générale de droit international public $1: 30-45$.

TAYLOR, T. 1995. Procureur à Nuremberg, Paris : Éditions du Seuil. 
TERNON Y. 1995. L'État criminel. Les Génocides au XX siècle, Paris : Seuil.

Traité de Lausanne, 24 juillet 1923, 28 R.T.S.N. 12.

Traité de Sèvres, 10 août 1920, 28 R.T.S.N. 223.

Traité de Versailles, 28 juin 1919, $\left(3^{\mathrm{e}}\right) 11$ MARTENS 323.

VerhoeVEN, J. 1991. «Le crime de génocide. Originalité et ambiguiité » Revue belge de droit international $1: 5-26$.

WECKEL, P. 1998. "La cour pénale internationale » Revue générale de droit international public 4 : 983-993.

WiEVIORKA, A. 1995. Le procès de Nuremberg, Rennes : Éditions Ouest-France.

ZOLLER, E. 1993. «La définition des crimes contre l'humanité » Journal du droit international $3: 549-568$. 